8'TH INTERNATIONAL SYMPOSIUM ON NONDESTRUCTIVE

CHARACTERIZATION OF MATERIALS, JUNE 15-20, 1997.

\title{
APPLICATION OF THE PSEUDO WIGNER-VILLE DISTRIBUTION TO THE MEASUREMENT OF THE DISPERSION OF LAMB MODES IN GRAPHITE/ EPOXY PLATES
}

\author{
W. H. Prosser ${ }^{1}$, M. D. Seale ${ }^{2}$, and B. T. Smith ${ }^{3}$ \\ ${ }^{1} \mathrm{MS} 231$ \\ ${ }^{2}$ Wheeling Jesuit University \\ NASA Langley Research Center \\ 316 Washington Ave. \\ Hampton, VA 23681-0001 \\ Wheeling, WV 26003 \\ ${ }^{3}$ Norfolk Academy \\ 1585 Wesleyan Drive \\ Norfolk, VA 23502
}

\section{INTRODUCTION}

Acoustic waves propagate in thin plates as guided or Lamb modes. The velocities of these modes are dispersive in that they depend not only on the material elastic properties and density, but also on the frequency. Accurate characterization of Lamb wave dispersion is important in many acoustic based nondestructive evaluation techniques. It is necessary for ultrasonic measurements in thin plates to determine elastic properties and for flaw detection and localization. In acoustic emission (AE) testing, if not taken into account, highly dispersive Lamb mode propagation can lead to large errors in source location ${ }^{1}$.

In this study, the pseudo Wigner-Ville distribution (PWVD) was used for measurement of group velocity dispersion of Lamb waves in a unidirectional graphite/epoxy (AS4/3502) laminate. The PWVD is one of a number of transforms which provide a time-frequency representation of a digitized time series. Broad band acoustic waves were generated by a pencil lead fracture (Hsu-Neilsen source) and were detected with broad band ultrasonic transducers. The arrival times for the lowest order symmetric $\left(\mathrm{S}_{0}\right)$ and antisymmetric $\left(\mathrm{A}_{0}\right)$ Lamb modes were determined from measurements of the time at which the respective peak amplitudes occurred in the PWVD. Measurements were made at several source-to-detector distances and a least squares fit used to calculate the velocity. Results are presented for propagation along, and perpendicular to, the fiber direction. Theoretical dispersion curves were also calculated and a comparison between theory and experiment demonstrates good agreement.

The PWVD and other time-frequency analyses offer two advantages for dispersion

measurements in comparison to other techniques. First, only a single measurement of a broad band signal is required to determine the velocity over a wide range of frequencies. Narrow 
band measurement techniques such as continuous wave resonant or tone burst pulse methods only provide measurements at a single frequency and require a large number of measurements to map out dispersion curves. The Fourier phase method is a velocity measurement technique that overcomes this problem using a broad band excitation ${ }^{7,8}$. The phase difference, as a function of frequency, between signals recorded at different distances of propagation is used to calculate dispersion. A difficulty arises in calculating the true phase which must be "unwrapped" to remove discontinuities when the calculated phase exceeds its $\pm \pi$ limits. A further problem with this and the previously mentioned narrow band techniques occurs when multiple propagating modes and/or reflections are superimposed in the time domain signal.

The second advantage of using time frequency analysis for dispersion measurement is that often the multiple modes or reflections can be separated in time-frequency space and such signals can still be analyzed. The reflection coefficient method can also analyze multiple mode signals and has been successfully used in Lamb wave dispersion measurements ${ }^{2-4}$. However, measurements must be repeated at multiple reflection angles and the plate must be immersed. The two-dimensional Fourier transform $\operatorname{method}^{5}$ can be used on signals containing multiple modes. In this approach, signals are detected at closely spaced intervals. A twodimensional Fourier transform is then applied which is used for phase velocity dispersion calculations. A large number of measurements are needed to avoid aliasing when Fourier transforming from the spatial domain. Other spectral estimation techniques such as Prony's method can be used to reduce this requirement for such high spatial sampling ${ }^{6}$.

\section{THEORY}

The general equation of a time-frequency distribution, $w(t, \omega)$ for signal $s(t)$ is given by

$$
w(t, \omega)=\frac{1}{2 \pi} \iiint e^{-i \theta t-i \tau \omega-i \theta u} \phi(\theta, \tau) s^{*}\left(u-\frac{\tau}{2}\right) s\left(u+\frac{\tau}{2}\right) d u d \tau d \theta
$$

where the integrals are evaluated from $-\infty$ to $\infty$. In this equation, $\mathrm{s}^{*}$ is the complex conjugate and $\phi(\theta, \tau)$ is an arbitrary function known as the kernel. Time and frequency are represented by $t$ and $\omega$ respectively ${ }^{9}$. For the Wigner distribution ${ }^{10-11}$, the kernel function has a value of 1. Substituting and applying to a sampled signal yields

$$
\mathrm{w}(\mathrm{m} \Delta \mathrm{t}, \mathrm{k} \Delta \omega)=2 \Delta \mathrm{t} \sum_{\mathrm{n}=0}^{2 \mathrm{~N}-1} \mathrm{~s}[(\mathrm{~m}+\mathrm{n}) \Delta \mathrm{t}] \mathrm{s}^{*}[(\mathrm{~m}-\mathrm{n}) \Delta \mathrm{t}] \mathrm{e}^{\frac{-\mathrm{i} 2 \pi \mathrm{nk}}{2 \mathrm{~N}}}
$$

where $\Delta \mathrm{t}$ is the sampling interval and $\Delta \omega=\pi /(2 \mathrm{~N} \Delta \mathrm{t})$. The Wigner distribution has several characteristics which limit its usefulness. The first is a higher sampling requirement to avoid aliasing. The sampling frequency must be four times that of the highest frequency content of the signal as opposed to the usual Nyquist criteria of only twice the highest frequency ${ }^{12}$. Additionally, the Wigner distribution produces complicated and unexpected results when more than one frequency component is contained in a signal. The Wigner distribution may contain signal amplitude at frequencies and times not actually contained in the original time signal. This artifact is caused by interference consisting of cross terms in the distribution from the multiple frequency components. It can make interpretation of results very difficult. The final undesirable characteristic of the Wigner distribution is that it may have negative values. These do not have physical meaning and most often occur as a result of this interference.

An approach to overcome the more restrictive sampling requirement was formulated by Ville $^{13}$. The Wigner-Ville distribution is calculated using analytic signal where the imaginary 
component is the Hilbert transform of the original time signal. The requirement to avoid aliasing is then reduced to that of the Nyquist criteria. An intuitive rational for this approach is based on the fact that a single sample of the analytic signal provides two effective samples (the real and imaginary parts) of the original signal ${ }^{12}$. Convolving a smoothing window with the distribution is used to minimize the effects of interference terms in the distribution and eliminate negative values. Smoothing, which results in the pseudo Wigner-Ville distribution, emphasizes deterministic components and reduces those due to interference ${ }^{11}$.

\section{EXPERIMENT}

Dispersion measurements were performed on a 16 ply unidirectional plate of AS4/3502 graphite/epoxy. The nominal plate thickness was $2.26 \mathrm{~mm}$ with lateral dimensions of 0.508 $\mathrm{m}$. along the fiber direction ( 0 degree direction) and $0.381 \mathrm{~m}$. along the 90 degree direction. Signals were generated by fracturing a $0.5 \mathrm{~mm}$. diameter pencil lead on the surface of the plate (Hsu-Neislen source). This source mechanism produces broad band, transient acoustic waves and is often used to simulate acoustic emission.

The acoustic waves were detected by a $3.5 \mathrm{MHz}$ ultrasonic sensor (Panametrics V182) which has a diameter of $1.27 \mathrm{~cm}$. This sensor is heavily damped. It was operated far off resonance in detecting these signals which had maximum frequency contents below $500 \mathrm{kHz}$. At these frequencies, this sensor provides a flat frequency, displacement sensitive response. The signals were digitized at a sampling frequency of $10 \mathrm{MHz}$ with 12 bit vertical resolution (Digital Wave Corporation F4012). The 2048 point waveforms were padded with 1024 zeros both in front of, and after the signal to increase the frequency resolution of the PWVD.

To improve accuracy, seven measurements were taken at different distances of propagation in $1.27 \mathrm{~cm}$. increments over a range of $8.89 \mathrm{~cm}$. to $16.51 \mathrm{~cm}$. A least squares fit of arrival time versus distance was used to calculate the velocity. The source position was kept fixed for all measurements and the receiver was moved to different positions. Waveform acquisition was triggered by a narrow band, $150 \mathrm{KHz}$ resonant sensor (Physical Acoustics Corporation R15) positioned adjacent to the source.

The original Fortran source code for the PWVD calculation from Jeon and Shin ${ }^{11}$ was modified for these dispersion measurements. The maximum number of points in the input signal and the maximum number of points in the calculated distribution were increased to provide enhanced time and frequency resolution. Peak detection routines were added to determine the arrival times of the two Lamb modes. A time separating the latest arrival of the $\mathrm{S}_{0}$ mode and earliest arrival of the $\mathrm{A}_{0}$ mode was determined the time domain signals and input into the program. At each frequency, the peak in the distribution before this input time value were used as the basis for the $S_{0}$ arrival times. The distribution peak after the input time value was used for the $\mathrm{A}_{0}$ arrival time. A seven point cubic spline fit was used to improve the resolution in determining the peak amplitudes (i.e. arrival times) of the two modes.

The PWVD results were displayed as grey-scale images. The grey level at a given $\mathrm{x}$ and y point in the image represents the amplitude of the distribution at a particular time and frequency. There was a large amplitude difference between the two modes. To more clearly show the two modes in the same image, the values of the distribution were compressed by taking the 4'th root which allowed adequate visualization of the data.

\section{RESULTS AND DISCUSSION}

Images of the PWVD along with the corresponding time domain signals for two distances of propagation are shown in Figure 1. The large dispersion of the $\mathrm{A}_{0}$ mode is clearly seen in both the time domain signals and the PWVD images. Higher frequencies arrive at 


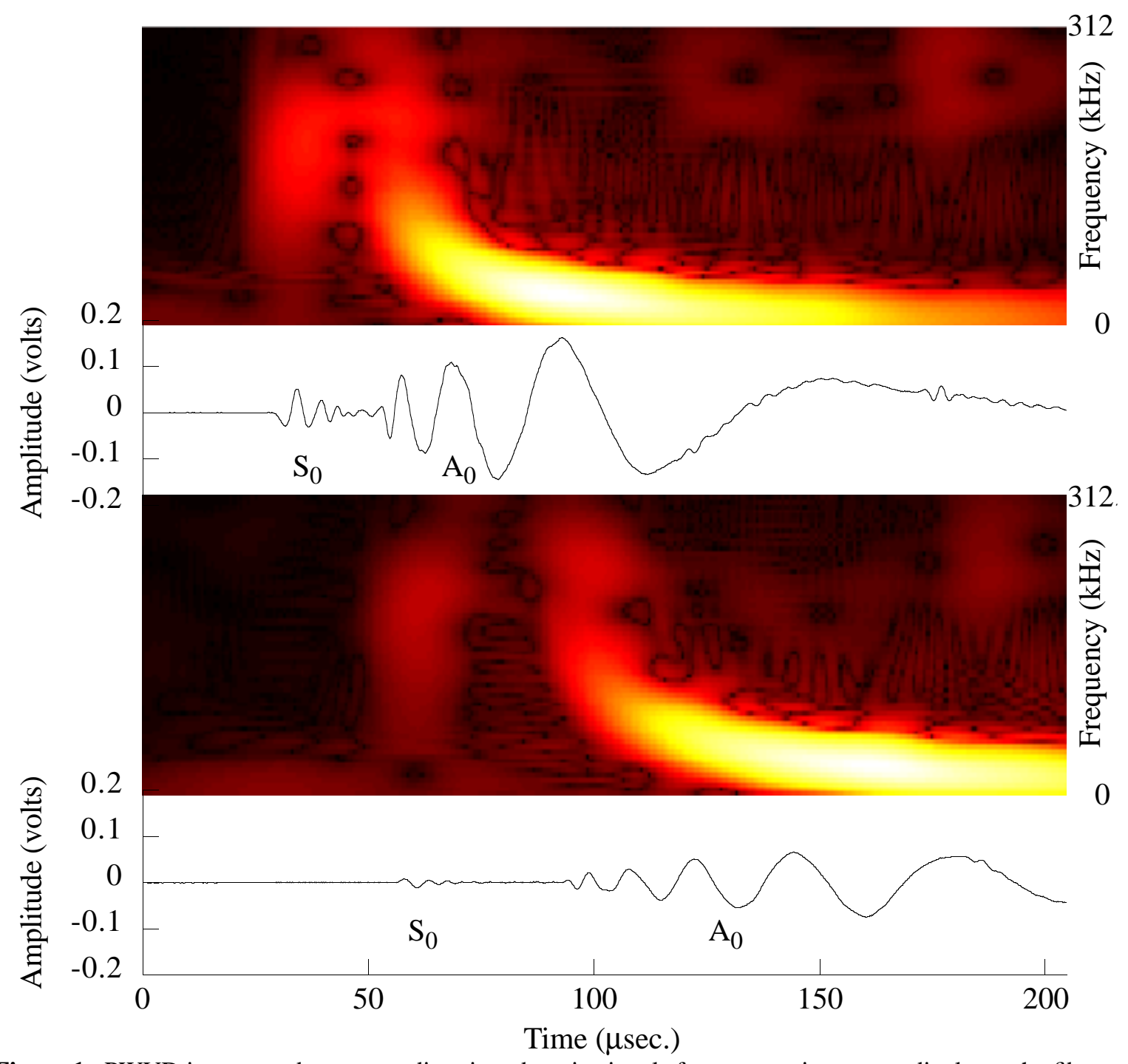

Figure 1. PWVD images and corresponding time domain signals for propagation perpendicular to the fiber direction at distances of $8.89 \mathrm{~cm}$. (upper) and $15.24 \mathrm{~cm}$. (lower).

earlier times in this mode and the signal changes shape as it propagates longer distances. Little dispersion is seen in the $S_{0}$ mode.

The measured dispersion results were compared with theoretical group velocity curves based on a finite element model ${ }^{14,15}$. Nominal elastic properties for a single lamina were obtained from the manufacturer and used in this calculation. These values are shown in Table 1 where the coordinate reference system is such that the 1 axis is along the fiber direction. The nominal density value of $1550 \mathrm{~kg} / \mathrm{m}^{3}$ was used.

The measured Lamb mode dispersion results for propagation perpendicular to the fiber direction are shown in Figure 2. The uncertainties in the measured velocity values as determined from the uncertainty of the slope in the least squares fit are displayed as error bars on the measured data. Comparison with theoretical curves show good agreement, particularly for the $A_{0}$ mode. The measured values for the $S_{0}$ mode are slightly higher than predicted. Similar results were obtained in phase velocity measurements in this material in an earlier study $^{8}$. A possible explanation for this slight discrepancy is that the material properties obtained from the manufacturer and used in the theoretical calculations are somewhat different from those of the actual material. Material property variations are not uncommon in graphite/epoxy and may be caused by fiber volume variations, differences in cure processing conditions, and variations in resin chemistry.

The results for propagation along the fiber direction are shown in Figure 3. Comparison with theoretical curves shows good agreement for the $A_{0}$ mode. The measured values of $S_{0}$ 


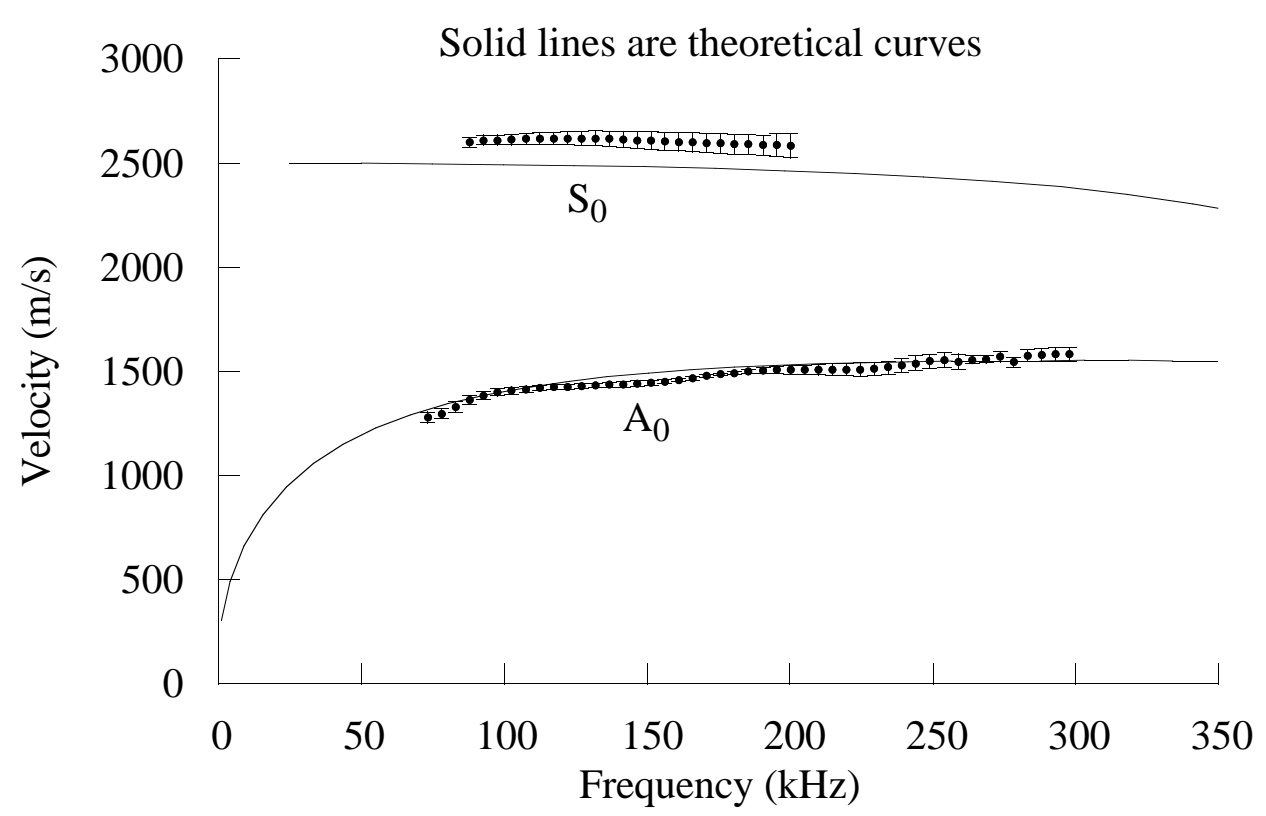

Figure 2. Measured Lamb mode group velocities along 90 degree propagation direction and comparison with theoretical predictions.

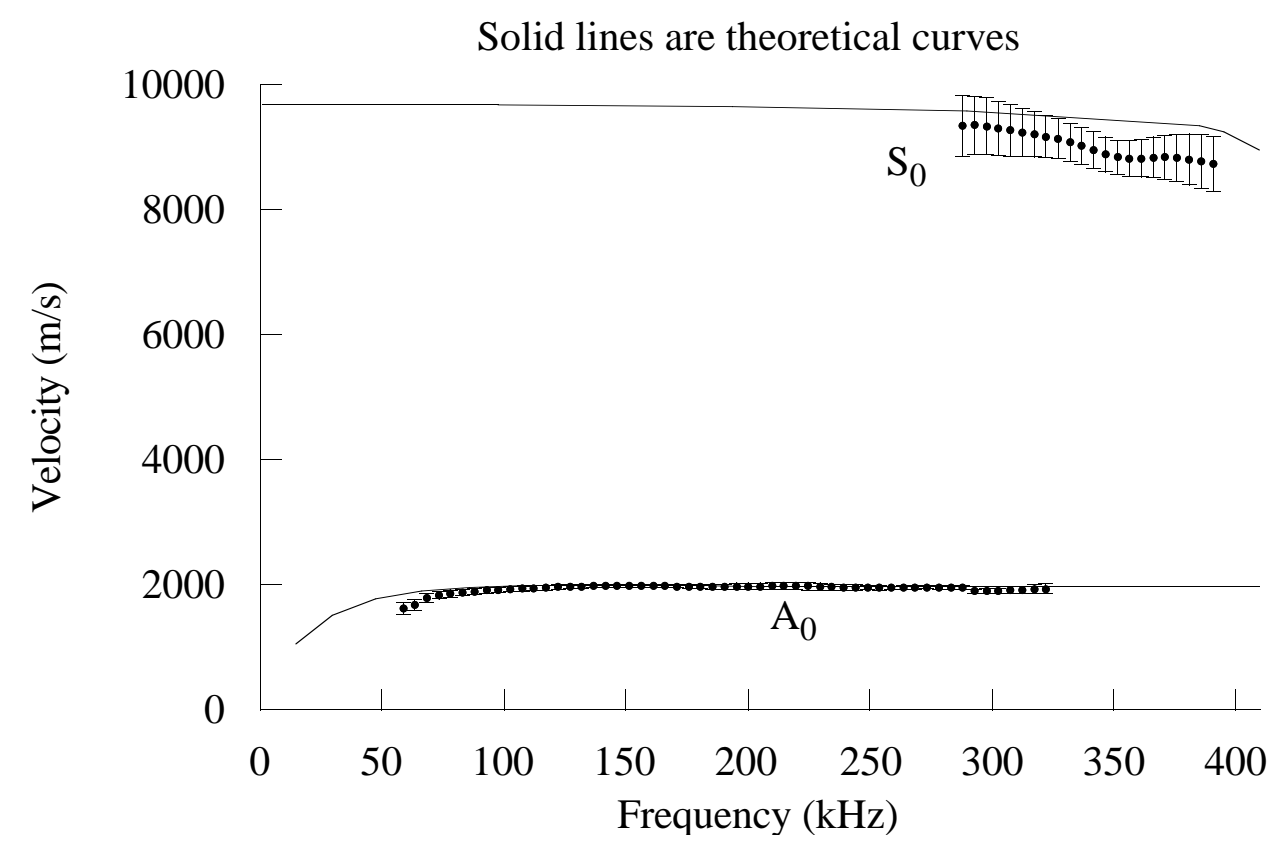

Figure 3. Measured Lamb mode group velocities along 0 degree propagation direction and comparison with theoretical predictions

mode are slightly lower than predicted along this direction. These results are again consistent with the previous phase velocity measurements.

In summary, a Pseudo Wigner-Ville distribution analysis has been used to measure the group velocity dispersion of Lamb waves in a unidirectional graphite/epoxy laminate. One advantage of this method is that the dispersion over a wide range of frequencies can be determined from a single measurement. Multiple measurements were used in this study to provide increased accuracy. Another advantage of time-frequency methods is that they can be used for signals which contain multiple modes. Results are presented for measurements both along, and perpendicular to the fiber direction. Comparison with theoretical predictions shows good agreement. The slight discrepancies between theory and experiment for the $\mathrm{S}_{0}$ mode velocities are consistent with previous phase velocity measurements in this material. 
Table 1. Nominal elastic moduli of AS4/3502 used in theoretical dispersion calculations.

\begin{tabular}{|c|c|c|c|}
\hline $\begin{array}{c}\text { Elastic } \\
\text { Modulus }\end{array}$ & $(\mathrm{GPa})$ & $\begin{array}{c}\text { Elastic } \\
\text { Modulus }\end{array}$ & $(\mathrm{GPa})$ \\
\hline \hline $\mathrm{c}_{11}$ & 147.1 & $\mathrm{c}_{22}$ & 10.59 \\
\hline $\mathrm{c}_{12}$ & 4.11 & $\mathrm{c}_{55}$ & 5.97 \\
\hline $\mathrm{c}_{23}$ & 3.09 & & \\
\hline
\end{tabular}

\section{REFERENCES}

1. S. M. Ziola and M. R. Gorman, Source location in thin plates using cross-correlation, JASA 90:2551-2556 (1991).

2. A. K. Mal, C. -C. Yin, and Y. Bar-Cohen, The influence of material dissipation and imperfect bonding on acoustic wave reflection from layered solids, Review of Progress in Quantitative Nondestructive Evaluation 7B:927-934 (1988).

3. D. E. Chimenti and A. H. Nayfeh, Leaky lamb waves in fibrous composite laminates, $J$. Appl. Phys. 58:4531-4538 (1985).

4. K. Balasubramaniam and J. L. Rose, Physically based dispersion curve feature analysis in the NDE of composites, Res. in NDE 3:41-67 (1991).

5. D. Alleyne and P. Cawley, A two-dimensional fourier transform method for the measurement of propagating multimode signals, JASA 89:1159-1168 (1991).

6. S. M. Kay and S. L. Marple, Jr., Spectrum analysis - a modern perspective, Proceedings of the IEEE 69:1380-1419 (1981).

7. W. Sachse and Y. H. Pao, On the determination of phase and group velocities of dispersive waves in solids, J. Appl. Phys. 49:4320-4327 (1978).

8. W. H. Prosser and M. R. Gorman, Plate mode velocities in graphite/epoxy plates, JASA 96:902-907 (1994).

9. L. Cohen, Time-frequency distributions - a review, Proceedings of the IEEE 77:941981 (1989).

10. E. Wigner, On the quantum correction for thermodynamic equilibrium, Physics Review 40:749-759 (1932).

11. J. Jeon and Y. S. Shin, Pseudo Wigner-Ville Distribution, computer program and its applications to time-frequency domain problems, Naval Postgraduate School Report NPS-ME-93-002 (1993).

12. B. Boashsah, Note on the use of the Wigner Distribution for time-frequency signal analysis, IEEE Transactions on Acoustics, Speech, and Signal Processing 36:15181521 (1988).

13. J. Ville, Theorie et applications de la notion de signal analytique, Cables et Transmission 2:61-74 (1948).

14. S. B. Dong and K. H. Huang, Edge vibrations in laminated composite plates, J. Appl. Mechs. 52:433-438 (1985).

15. S. K. Datta, A. H. Shah, and W. Karunasena, Wave Propagation in Composite Media and Material Characterization, Elastic Waves and Ultrasonic Nondestructive Evaluation, S.K. Datta, J.D. Achenbach, and Y.S. Rajapakse, eds., Elsevier Science Publishers B.V., North-Holland (1990). 